\title{
2017 Thank you to our reviewers
}

The Editor would like to publicly acknowledge the people listed below who served as reviewers on the journal during 2017. Without their efforts, the quality of the journal could not be sustained.

\author{
Anderson, Jane \\ Anderst, Leah \\ Atilola, Olayinka \\ Atkinson, Sarah \\ Ayala, Ricardo \\ Baelo-Allue, Sonia \\ Barclay, Katie \\ Bardill, Jessi \\ Beaney, Thomas \\ Berkowitz, Carin \\ Beveridge, Allan \\ Billington, Josie \\ Blanton, Sarah \\ Bleakley, Alan \\ Bolaki, Stella \\ Buchman, Daniel \\ Burke, Lucy \\ Burkle, Frederick M \\ Carmel-Gilfilen, Candy \\ Carrieri, Daniele \\ Cerame del Campo, Alvaro \\ Chen, Julie \\ Classen, Albrecht \\ Comoretto, Nunziata \\ Cook-Deegan, Robert \\ Courneya, Carol Ann \\ Cox, Susan \\ Cupitt, Caroline \\ Davis, Mark D M \\ Day, Giskin \\ Day, Sophie \\ Diamond, Randi
}

Dingle, Genevieve

Dolezal, Luna

Donahoo, Robert

Downer, Simon

Dryer, Marc

Dyck, Erika

Foley, Ronan

Fox, Nick

Freidenfelds, Lara

Fullagar, Simone

Garrisi, Diana

Godderis, Rebecca

Goldberg, Daniel

Graham, Jeremy

Harpin, Anna

Hayes, Nick

Hopkins, Stephen

Houghton, Miriam

Houston, Muiris

Howard, Fuchsia

Jansson, Asa

Jenner, Mark

Johnson, Bethany

Jones, David

Jones, Nev

Kaliyadan, Feroze

Kaptein, Ad

Kemp, Sandra

Kidd, David Comer

Knellwolf King, Christa

Kumagai, Arno

Lafrance, Marc
Lapum, Jennifer

Lowrey, Belen V

Lyons, Barry

Lyons, Zaza

Maatz, Anke

Martel, Sara

McAtackney, Laura

McCarthy-Jones, Simon

McLaren, Coralee

Meek, Heather

Meloncon, Lisa

Millar, Bonnie

Murray, Stuart

Newton-Howes, Giles

$\mathrm{Ng}$, Derek

Nowaczyk, Malgorzata

O'Neill, Desmond

Palfreyman, Harriet

Pattison, Stephen

Paz-Lourido, Berta

Peacock, Marian

Piggford, George

Pryjmachuk, Steven

Reid, Cary

Reid, Lindsay

Reynolds, Joel

Robbins, Gina

Robinson, Lucy

Ruston, Sharon

Şahinol, Melike

Salice, Alessandro

Salisbury, Laura
Salzer, Mark S

Sandassie, Samantha

Schwind, Jasna

Shale, Suzanne

Shih, Patti

Sidlauskas, Susan

Smith, Lisa

Sneddon, Andrew

Sparrow, Robert

Spiegel, Maura

Squier, Susan

Stafford, Anika

Tan, Jacinta

Tariq, Shema

Thomas, Philip

Thoresen, Lisbeth

Vaccarella, Maria

van der Zwart, Johan

Van Lith, Theresa

Viney, William

Walbaum, Sharlene

Walitt, Brian

Warner, Catherine

Wasson, Sara

Webb, Jen

Weisser, Olivia

Wellbery, Caroline

Whitehead, Anne

Wolf, Jacqueline

Woods, Angela

Younie, Louise

Zambrano, Sofia C 